\title{
MUCINOUS BILIARY CYSTADENOMA OF THE LIVER MIMICKING A HYDATID CYST
}

\author{
F. Limaiem ${ }^{1}$ and saadia bouraoui ${ }^{1}$ \\ ${ }^{1}$ University Hospital Center Mongi Slim
}

October 1,2020

\begin{abstract}
Mucinous cystic neoplasm of the liver is a rare slow-growing lesion accounting for less than $5 \%$ of all hepatic cystic neoplasms. Differential diagnosis from other cystic lesions remains challenging despite progress achieved in the radiological modalities. Only histopathological examination of the surgical specimen establishes with certainty the diagnosis.
\end{abstract}

\section{CLINICAL IMAGE}

A 40-year-old female patient with a past medical history of hypothyroidism, presented with a four-month history of right upper quadrant pain and weight loss. Abdominal computed tomography scan revealed a $73.75 \times 48.38 \mathrm{~mm}$ unilocular cystic mass in the right hepatic lobe suggestive of hydatid cyst (Fig. 1A). Laboratory tests were within normal limits. Tumor markers (including CA19-9) and serum IgE level were not performed. Given her history of proximity to livestock in an endemic area, the diagnosis of a hydatid cyst was strongly favored. However, echinococcosis serology was negative. The patient underwent a right hepatectomy through a right subcostal incision. Grossly, the cystic mass was surrounded by a thick whitish capsule and contained a clear mucinous fluid (Fig 1B). Histological examination demonstrated a cystic lesion lined by a columnar biliary type epithelium overlying dense spindled ovarian type stroma (Figures 1C and 1D). There were signs of cytological atypia or mitoses. The final pathological diagnosis was mucinous biliary cystadenoma of the liver. The postoperative course was uneventful. At present, the patient is still being followed up.

\section{CONFLICT OF INTEREST STATEMENT}

None declared.

\section{AUTHORS CONTRIBUTIONS}

Dr Faten LIMAIEM prepared, organized, wrote, and edited all aspects of the manuscript. She performed the gross and microscopic pathologic evaluation of the pathology specimen. She prepared all of the histology figures in the manuscript. She read, edited, and approved the final version of the manuscript. Pr Saadia BOURAOUI participated in the drafting of the article and revising it critically for important intellectual content,

\section{ACKNOWLEDGEMENT STATEMENT}

I would like to offer my special thanks to Mrs Saïda Jerbi who prepared the microscopic slides.

\section{REFERENCES}


[1] Zen Y, Pedica F, Patcha VR, Capelli P, Zamboni G, Casaril A. 2011. Mucinous cystic neoplasms of the liver: a clinicopathological study and comparison with intraductal papillary neoplasms of the bile duct. Modern Pathology 24: 1079-89.

[2] Averbukh LD, Wu DC, Cho WC, Wu GY. 2019. Biliary Mucinous Cystadenoma: A Review of the Literature. J Clin Transl Hepatol 28; 7(2): 149-53.

\section{FIGURE LEGENDS:}

\section{MULTIPANEL FIGURE LEGEND:}

Figure 1A : Axial CT scan demonstrating a unilocular cystic mass within the right hepatic lobe of the liver, measuring $73.75 \times 48.38 \mathrm{~mm}$.

Figure 1B: Macroscopic findings of mucinous cystadenoma. A unilocular well-delineated cystic mass of the liver surrounded by a thick fibrous capsule.

Figure 1C: Solitary cystic lesion of the liver lined by simple epithelium overlying ovarian type stroma (Hematoxylin and eosin, magnification $\times 40$ ).

Figure 1D: Histological examination of the hepatic cyst showing columnar biliary type epithelium overlying dense spindled ovarian type stroma (Hematoxylin and eosin, magnification $\times 400$ ).

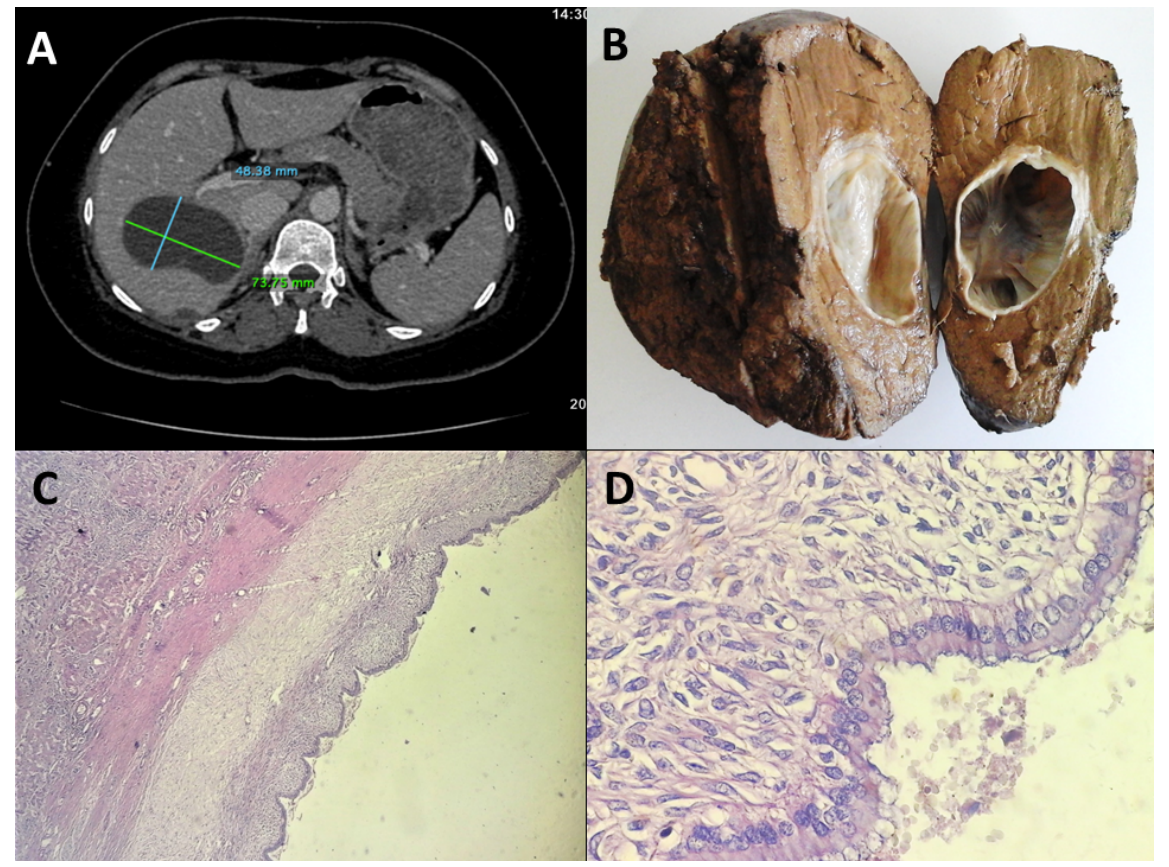

